\title{
Diabetes y hemodiálisis. Caracterización de una cohorte y seguimiento a cuatro años
}

\author{
María Eugenia Sanhueza ${ }^{1}$, Alejandro Cotera ${ }^{1}$, \\ Leticia Elgueta ${ }^{1}$, G loria López $S^{2}$, Patricia Loncon4a, \\ Fernando Macan ${ }^{b}$, Francisco Pérez ${ }^{\text {b }}$, Gabriel Cavada ${ }^{3 c}$, \\ Miriam Alvo ${ }^{1}$.
}

\section{Assessment and follow up of diabetic patients in hemodialysis}

Background: Despite a better management of the variables that influence the development of diabetic nephropathy, there is a progressive increase in the prevalence of terminal renal failure among diabetics, whose cause is not clear. Aim: To study, in a group of patients in hemodialysis, the quality of diabetes control previous to the entry to dialysis, their physical condition and their evolution. Material and methods: Diabetic patients with at least three months of hemodialysis answered a questionnaire about diabetes control quality previous to dialysis and had physical and laboratory assessment. They were followed for at least four years thereafter. Results: Fifty seven patients aged $62 \pm 11$ years were studied. Eighty four percent had some degree of disability. Eighty seven percent had high blood pressure and $73 \%$ had to enter dialysis as an emergency. Mean glycosilated hemoglobin was $7.7 \%$ and $58 \%$ had a dialysis dose with a Kt/V of less than 1.2. Fifty eight percent died during follow up. No relationship between mortality and age, blood pressure, glycosilated hemoglobin of $\mathrm{Kt} / \mathrm{V}$, was observed. Conclusions: There is an inadequate management of blood glucose and blood pressure of diabetic patients before entry to dialysis. They are referred late to the nephrologist, the dialysis dose is insufficient and they have a high mortality (Rev Méd Chile 2008; 136: 279-86).

(Key words: Diabetes complications; Kidney failure, chronic; Renal dialysis)

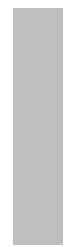

Recibido el 19 de junio, 2007. Aceptado el 28 de septiembre, 2007.

Secciones de Nefrología y Endocrinología ${ }^{2}$, Departamento de Medicina, Hospital Clínico Universidad de Chile. ${ }^{3}$ Escuela de Salud Pública, Facultad de Medicina, Universidad de Chile. Santiago de Chile. ${ }^{4}$ Hospital Clínico de la Universidad de Chile.

${ }^{\text {a Nutricionista }}$

bAlumnos de Medicina

${ }^{\mathrm{c}}$ Bioestadístico

$\mathrm{L}$ a diabetes representa la principal causa de insuficiencia renal crónica en la mayor parte

Correspondencia a: Dra. María Eugenia Sanhueza Villanueva. Santos Dumont 999, Independencia. Fax: 7352964. E mail: hemofiltracion@yahoo.es/nefrolog@redclinicauchile.cl de los países del mundo ${ }^{1-4}$. El desarrollo de la nefropatía diabética depende de factores genéticos, del adecuado control glicémico, de cifras de presión arterial y del uso de fármacos que específicamente detienen e incluso revierten nefropatías clínicas iniciales $^{5-8}$. A pesar del evidente progreso en la comprensión y manejo de las variables que 
influyen en el desarrollo de la nefropatía diabética, se ha demostrado un aumento progresivo de la prevalencia de insuficiencia renal crónica terminal en pacientes diabéticos, cuya causa no ha sido determinada? 9 . Por otra parte, los pacientes con nefropatía diabética tienen una morbimortalidad muy superior a los pacientes con insuficiencia renal de otras causas, que es atribuida a la comorbilidad y en parte a factores propios de la diabetes ${ }^{1,10,11}$. El propósito del presente trabajo fue estudiar, en una población de pacientes diabéticos en hemodiálisis, factores relacionados al cuidado prediálisis, comorbilidad, capacidad funcional, estado nutricional, modo de ingreso a diálisis y factores asociados a la calidad de diálisis $\mathrm{y}$ analizar la sobrevida, causas de muerte y hospitalización, durante un periodo de seguimiento de cuatro años.

\section{Material y MÉTOdo}

En mayo de 1999, se realizó un censo de todos los pacientes en hemodiálisis del área norte de Santiago y se seleccionaron aquellos en los que la causa de la enfermedad renal terminal era la diabetes y que llevaban a lo menos tres meses en diálisis. A los pacientes se les aplicó una encuesta especialmente diseñada para evaluar la calidad de control en pacientes diabéticos (Qualidiab) ${ }^{12}$, a la que se le agregan los datos referentes a hemodiálisis. Los aspectos que explora la encuesta se muestran en la Tabla 1. El grado de discapacidad física se estimó mediante el índice de Karfnofsky ${ }^{13}$. Se realizó una evaluación nutricional que incluyó índice de masa corporal, antropometría (perímetro braquial y pliegue tricipital) y medición de albúmina sérica. El control metabólico se evaluó con hemoglobina glicosilada (HbA1C), la que se determinó mediante captura iónica. De los registros de diálisis se obtuvieron datos referentes a la condición al ingreso a diálisis, se definió como urgencia el ingreso a diálisis en síndrome urémico, edema pulmonar agudo o hiperkalemia; acceso vascular, las cifras de presión arterial prediálisis de mediado de semana de los últimos 3 meses y evaluaciones de laboratorio, que son el promedio de tres muestras en meses sucesivos. La dosis de la diálisis se estimó mediante el índice Kt/V usando la fórmula de Dauguirdas II $^{14}$ y mediante el porcentaje de reducción de la urea durante la sesión de diálisis.

Los pacientes fueron seguidos durante 4 años, se registraron los datos relacionados a las hospitalizaciones, fallecimientos y causas de muerte. Todos los pacientes entregaron su consentimiento informado para participar en el estudio.

Tabla 1. Encuesta Q ualidiab modificada

1. Datos demográficos

2. Diabetes

Tiempo de evolución

Tipo de diabetes

Tipo de control

Tratamiento

3. Nefropatía

Fecha del diagnóstico

Tratamiento

4. Evaluación oftalmológica

Evaluación previa

Retinopatía

5. Evaluación de los pies

Evaluación previa

Amputaciones
6. Evaluación cardiovascular

Angina

Infarto

Cirugía de revascularización

Accidente cerebrovascular

Claudicación intermitente

Impotencia

7. Tratamiento y control actual de la diabetes

Asistente a control

Control glicémico/autocontrol

Tratamiento

8. Diálisis

Fecha de ingreso

Modo de ingreso

Acceso vascular 
Estadística. Las variables cuantitativas se expresan como promedio \pm desviación estándar. En las comparaciones se utilizó el test t de Student. Las variables categóricas se describen mediante tablas de frecuencia y se comparan usando el test de independencia de $\mathrm{Chi}^{2}$ y test exacto de Fisher. La función de sobrevida se describe mediante el método de Kaplan-Meier, las curvas de sobrevida se comparan usando el test de Log rank y se construye un modelo multivariado de sobrevida usando regresión de Cox. Los intervalos de confianza son de nivel de $95 \%$ y la significación de los test de $5 \%$.

\section{RESUlTADOS}

Al momento del censo habían 344 pacientes en programa de hemodiálisis en el área norte de Santiago, de éstos, 57 (16,6\%) eran diabéticos, en que la causa de la enfermedad renal crónica presumiblemente era la nefropatía diabética. Sólo 3 pacientes eran diabéticos tipo 1 y 54 tipo 2 $(94,7 \%)$. La edad promedio de los pacientes fue de $62 \pm 10,6$ años. Las otras características epidemiológicas y demográficas de estos pacientes se muestran en la Tabla 2 y el de los análisis de laboratorio en la Tabla 3. El tiempo promedio desde el ingreso a diálisis al reclutamiento para el estudio fue de 20,0 $\pm 20,9$ meses. El $84 \%$ de los pacientes tenían algún grado de discapacidad al momento de la encuesta. El tiempo de evolución conocido de la diabetes era de 17,9 $\pm 11,1$ años y el de la hipertensión arterial 8,7 $\pm 9,5$. El diagnóstico de la nefropatía diabética fue conocido por los pacientes $10,7 \pm 2$ meses previo al ingreso a

Tabla 2. Características demográficas y epidemiológicas

\begin{tabular}{|ll|}
\hline Pacientes diabéticos & $57(16,7 \%)$ \\
DM tipo I & $3(5,3 \%)$ \\
DM tipo II & $54(94,7 \%)$ \\
Sexo & $30(52,6 \%)$ \\
Masculino & $27(47,4 \%)$ \\
Femenino & $62 \pm 10,6$ años \\
Edad & $17,9 \pm 11,1$ años \\
Tiempo de evolución de DM & \\
\hline
\end{tabular}

diálisis. La diabetes fue controlada en un programa estructurado en $49,1 \%$ de los pacientes. Previo al ingreso a diálisis, $66 \%$ de los pacientes recibía exclusivamente hipoglicemiantes orales y $14 \%$ insulina sola o en combinación con hipoglicemiantes orales. Antes del ingreso a diálisis, 80,7\% de los pacientes conocían su condición de hipertenso, de éstos, 91,3\% recibían tratamiento farmacológico, el que en 14,3\% incluía inhibidores de la enzima convertidora de angiotensina. El 82,5\% de los pacientes había tenido control oftalmológico, $66,7 \%$ padecía algún grado de ambliopía y $12,3 \%$ amaurosis. El 12,3\% de los pacientes tenía alguna amputación en las extremidades inferiores y $42 \%$ había recibido evaluación del estado de sus pies. El 26\% de los pacientes tenía evidencia clínica de cardiopatía coronaria, que estaba constituida por angina en $85,7 \%$, antecedente de infarto agudo del miocardio en $42,6 \%$ y de revascularización en $28,6 \%$. Otros resultados de la evaluación del estado cardiovascular se presentan en la Tabla 4.

Según los índices antropométricos, 63,2\% de los pacientes eran eutróficos y 19,3\% obesos o con sobrepeso. La HbA1C promedio fue de 7,7 \pm $1,7 \%$, siendo menor de 6,5 en $32,7 \%$, y mayor de 8,0 en $32,7 \%$ de los pacientes.

El ingreso a diálisis fue en condición de urgencia en $73 \%$ de los pacientes. Al momento de la encuesta, 93,0\% de los pacientes tenía un acceso vascular definitivo que en $79,2 \%$ era una fístula arteriovenosa nativa. La presión arterial

\section{Tabla 3. Exámenes de laboratorio} de pacientes diabéticos en H D al ingreso al estudio

\begin{tabular}{|lr|}
\hline Hematocrito & $28 \pm 4,4 \%$ \\
Calcemia & $8,9 \pm 0,57 \mathrm{mg} / \mathrm{dl}$ \\
Fósforo & $4,9 \pm 1,35 \mathrm{mg} / \mathrm{dl}$ \\
Producto calcio fósforo & 46,7 \\
Potasio & $5,3 \pm 0,6 \mathrm{meq} / \mathrm{l}$ \\
Albúmina & $4,4 \pm 0,5 \mathrm{~g} / \mathrm{l}$ \\
N.U prediálisis & $68 \pm 14,8 \mathrm{mg} / \mathrm{dl}$ \\
N.U postdiálisis & $27 \pm 8,1 \mathrm{mg} / \mathrm{dl}$ \\
URR & $0,62 \pm 0,05$ \\
Kt/V & $1,17 \pm 0,16$ \\
Hemoglobina glicosilada & $7,7 \pm 1,7 \%$ \\
\hline
\end{tabular}

N.U.: nitrógeno ureico. URR: tasa de reducción de urea. $\mathrm{Kt} / \mathrm{V}$ : dosis de diálisis. 
Tabla 4. Evaluación del estado cardiovascular al ingreso al estudio

\begin{tabular}{|c|c|}
\hline & Número de pacientes \\
\hline Cardiopatía coronaria & $14 \quad(24,6 \%)$ \\
\hline Angina & 12 \\
\hline Infarto al miocardio & 6 \\
\hline Cirugía de revascularización & 4 \\
\hline Claudicación intermitente & $18 \quad(31,6 \%)$ \\
\hline Accidente cerebrovascular & $4 \quad(7 \%)$ \\
\hline Hipotensión postural & $20 \quad(35,1 \%)$ \\
\hline Disfunción eréctil & $25 \quad(80,3 \%)$ \\
\hline
\end{tabular}

prediálisis promedio de tres sesiones era $160 \pm 15$ mmHg de sistólica con $85 \pm 7 \mathrm{mmHg}$ de diastólica. El $87,7 \%$ de los pacientes tenían presión arterial mayor de $140 \mathrm{mmHg}$ de sistólica o $90 \mathrm{mmHg}$ de diastólica, 41\% tenían hipertensión diastólica.

$\mathrm{El} \mathrm{Kt} / \mathrm{V}$ promedio fue de $1,17 \pm 0,16$, en $57,9 \%$ de los pacientes fue menor de 1,2. Otros parámetros de la calidad de diálisis se presentan en la Tabla 3.

Al momento de la reevaluación de esta cohorte, realizada a los cuatro años del reclutamiento, 33 de los 57 pacientes $(57,8 \%)$ habían fallecido, la sobrevida a los 12 y 48 meses fue de $84 \%$ y $42,2 \%$, respectivamente (Figura 1). La causa de muerte fue cardiovascular en $35 \%$ e infecciosa en $28 \%$, hubo tres casos (11\%) de muertes en relación a hipoglicemia (Figura 2). No hubo diferencias entre los fallecidos y los sobrevivientes en cuanto a edad, presión arterial, hemoglobina glicosilada, $\mathrm{Kt} / \mathrm{V}$, hematocrito o fósforo. El $74 \%$ de los pacientes se hospitalizó alguna vez durante el seguimiento, con un total de 173 hospitalizaciones. Los sobrevivientes tuvieron una tasa de hospitalizaciones de 0,59 hospitaliza-

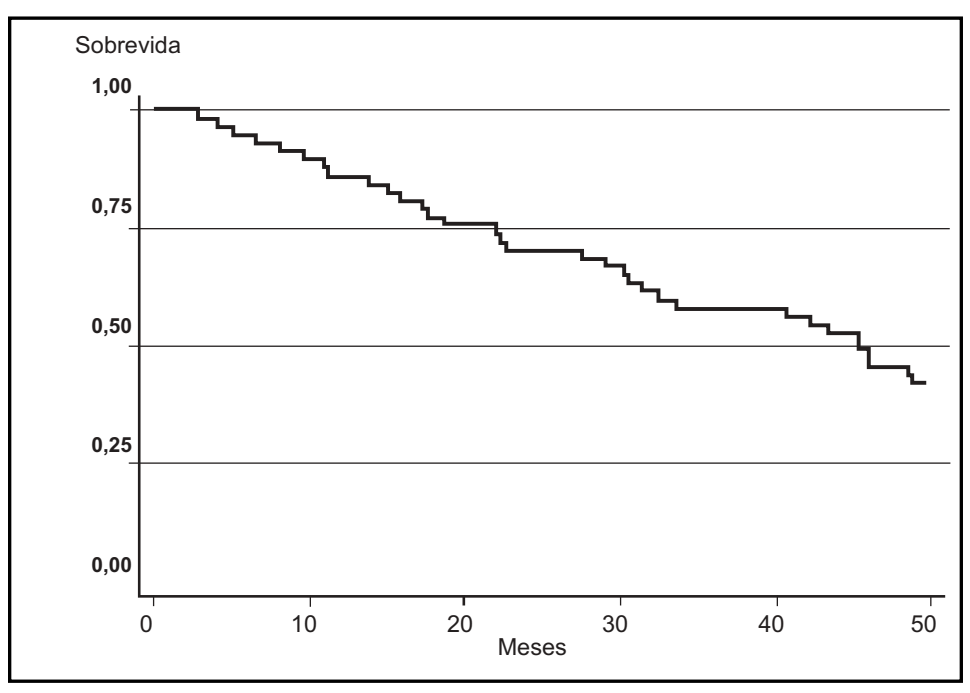

Figura 1. Sobrevida de pacientes diabéticos en hemodiálisis. La curva de Kaplan-Meier muestra la sobrevida de los pacientes diabéticos en hemodiálisis durante un seguimiento a cuatro años. 
ciones/paciente/año y los fallecidos 1,0 hospitalizaciones/paciente/año ( $p<0,04)$. Las causas de las hospitalizaciones se muestran en la Figura 3.

\section{DisCUSIÓN}

La diabetes mellitus es la primera causa de insuficiencia renal crónica en el mundo y en
Chile $e^{1-4,15}$. La insuficiencia renal secundaria a diabetes ha sido recientemente calificada como una epidemia en Estados Unidos de Norteamérica? ${ }^{9}$ Los pacientes diabéticos en hemodiálisis tienen una elevadísima morbimortalidad, la que parece estar relacionada con el cuidado prediálisis, la comorbilidad y el cuidado de los pacientes durante la diálisis ${ }^{1,10-11}$. Éste es el primer estudio que investiga las características y evolución de pacientes

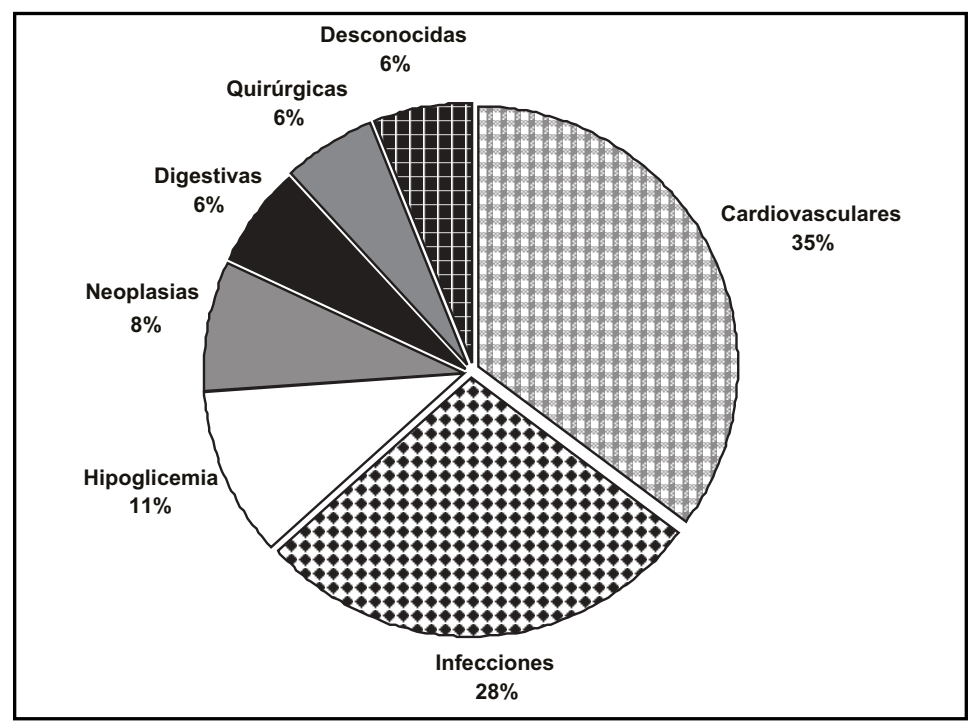

Figura 2. Causas de muerte de pacientes diabéticos en hemodiálisis durante el seguimiento a cuatro años.

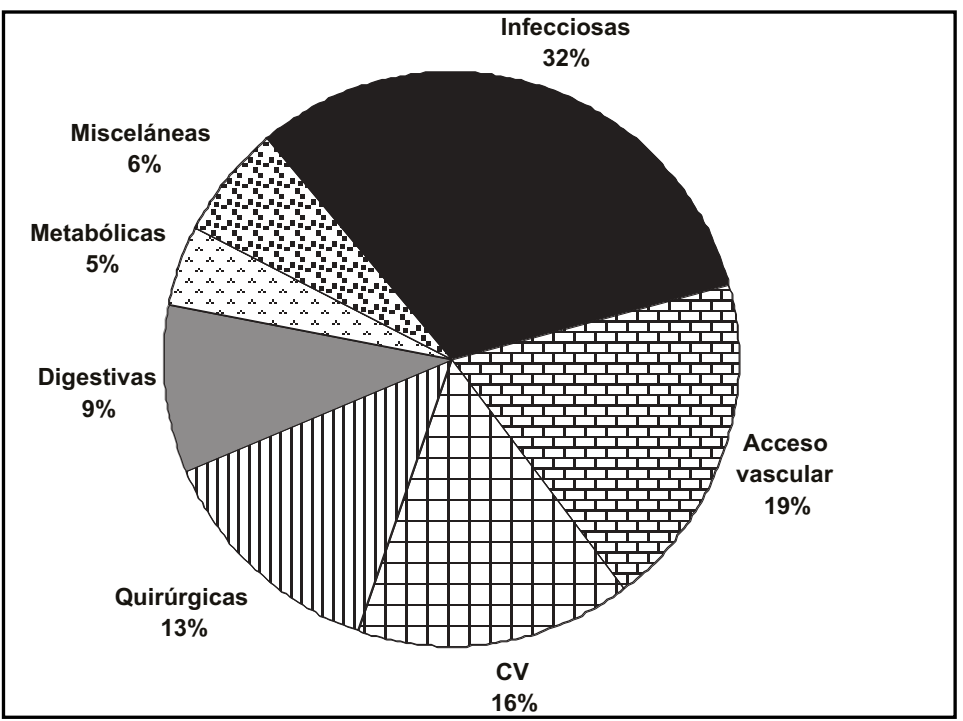

Figura 3. Hospitalizaciones de pacientes diabéticos en hemodiálisis durante el seguimiento a cuatro años. 
diabéticos en hemodiálisis en Chile, donde se exploran los factores relacionados con el manejo predialítico, comorbilidad, modo de ingreso a diálisis, control metabólico de la diabetes, dosis de diálisis, y evolución durante un seguimiento de 4 años, de la población de pacientes diabéticos en hemodiálisis en el área norte de Santiago. En mayo de 1999 habían 57 pacientes diabéticos en diálisis, que constituían 16,6\% del total de los pacientes en hemodiálisis en el área norte de Santiago, esta cifra es menor a lo reportado en el país en ese período, en que los pacientes diabéticos representaban $26,7 \%$ de los pacientes en diálisis ${ }^{16}$. Esta discrepancia puede tener variadas explicaciones como: sesgo por la referencia de los pacientes diabéticos a otros servicios de salud, restricción del ingreso a diálisis de los pacientes diabéticos, menor prevalencia de diabetes o nefropatía diabética en el área estudiada, mayor mortalidad de los diabéticos en la etapa previa al ingreso a diálisis o en los primeros 90 días de hemodiálisis.

La relación entre pacientes diabéticos tipo 1 y 2 en diálisis es consistente con la frecuencia de las dos formas de la enfermedad reportada en Chile $\mathrm{e}^{17,18}$, lo que difiere con la realidad de otros países en que la frecuencia de la diabetes tipo 1 es más alta y representa hasta $30 \%$ de los diabéticos en diálisis ${ }^{19-21}$.

El tiempo de evolución conocida de la enfermedad, 17,9 años, es concordante con lo que sabemos sobre la evolución de la nefropatía en la diabetes mellitus ${ }^{22}$. Los pacientes conocieron el diagnóstico de nefropatía diabética en promedio $10,7 \pm 2$ meses antes del ingreso a diálisis y $73 \%$ de los pacientes ingresó a diálisis en condición de emergencia, esto refleja un retardo en el diagnóstico de la nefropatía y en la referencia al especialista, lo que reviste particular importancia, dado que tanto la referencia tardía al nefrólogo como el ingreso a diálisis en condición de emergencia, empeoran el pronóstico de los pacientes en diálisis. Sin embargo, es importante destacar que esto también ocurre en otros países, incluso en aquellos de mayor desarrollo económico $23-25$.

En la cohorte estudiada destaca que sólo la mitad de los pacientes había tenido control en un programa estructurado de diabetes previo al ingreso a diálisis, a pesar de la existencia de un programa que estimaba una cobertura de 95,9\% en el área norte de Santiago en junio de 1998 (Ministerio de Salud). Esto, posiblemente, contribuye a un inadecuado control metabólico y a una insuficiente prevención, detección y tratamiento de las complicaciones, lo que condiciona el ingreso a diálisis en situación de emergencia de pacientes con una importante carga de morbilidad. En el grupo de pacientes estudiado, 66\% tenía algún grado de ambliopía, 12\% de amaurosis, $24 \%$ evidencia de cardiopatía coronaria clínica y $12 \%$ de amputaciones; todos estos están relacionados fuertemente a mayor mortalidad precoz luego del ingreso a diálisis ${ }^{23-26}$.

El control metabólico, evaluado a través de la hemoglobina glicosilada al momento del ingreso al estudio, resultó ser bastante insuficiente. La hemoglobina glicosilada, en promedio fue de $7,7 \pm$ $1,2 \%$, siendo mayor a $8 \%$ en un tercio de los pacientes. Una realidad semejante ha sido reportada por Oomichi ${ }^{27}$ en Japón, quien también estudió el efecto del control de la glicemia de pacientes que ya se encontraban en hemodiálisis mediante hemoglobina glicosilada, y demostró que valores de hemoglobina glicosilada superior a $8 \%$ condicionan una mayor mortalidad de los pacientes en diálisis. La exploración del tratamiento farmacológico mostró que $78 \%$ de nuestros pacientes recibían hipoglicemiantes orales (predominantemente glibenclamida) solos o asociados a insulina, indicación que en pacientes en insuficiencia renal debería ser muy restringida debido al elevado riesgo de hipoglicemia, esto está en abierto contraste a lo que sucede en Estados Unidos de Norteamérica en que el tratamiento farmacológico de la diabetes en hemodiálisis es casi exclusivamente insulina ${ }^{28}$.

El $76,7 \%$ de los pacientes tenían cifras de presión arterial mayor de $140 \mathrm{mmHg}$ de sistólica o $90 \mathrm{mmHg}$ de diastólica, la elevada frecuencia de hipertensión arterial es semejante a lo que ha sido publicado por Vukusich en los pacientes diabéticos chilenos en diálisis ${ }^{29}$.

La adecuación de la diálisis, medida como la extracción de urea en relación al volumen de distribución de ésta, Kt/V, ha demostrado estar directamente relacionada a la morbilidad y mortalidad de los pacientes en diálisis, un Kt/V de a lo menos 1,2 es la recomendación internacional ${ }^{30}$. En nuestros pacientes, al momento de ingreso al estudio, el Kt/V promedio fue de 1,17 con $57 \%$ de los pacientes con dosis de diálisis por debajo de la 
recomendación. Ese año, en Chile, en la XIX Cuenta de Hemodiálisis, Poblete ${ }^{16}$ comunicó que $51,6 \%$ de los pacientes en Chile recibían un Kt/V menor de 1,2, lo que sugiere que en esta cohorte la dosis de diálisis era semejante a la de los pacientes no diabéticos.

Durante el seguimiento de 4 años falleció el $57,8 \%$ de los pacientes, siendo la mortalidad de $16 \%$ el primer año. Es conocido que los pacientes diabéticos tienen una elevada mortalidad en relación a pacientes no diabéticos ${ }^{31}$, la cifra de mortalidad bruta al año encontrada en el presente estudio es semejante a la reportada en la encuesta nacional de diálisis para el mismo periodo ${ }^{16}$, en contraste, la población de dializados no diabéticos mostró una mortalidad de sólo 7,5\% en Chile en el mismo periodo. No contamos con datos nacionales para comparar la mortalidad a plazos mayores de un año. Con respecto a las causas de muerte, las principales fueron la cardiovascular $35 \%$ e infecciosas $28 \%$, lo que es semejante a lo descrito en la literatura ${ }^{1}$. Destacan tres muertes en relación a hipoglicemia, lo que debe alertarnos a la complejidad del manejo glicémico en estos pacientes. No se encontró asociación de la mortalidad con varios factores que incluyen edad, sexo, cifras de presión arterial, hematocrito, hemoglobina glicosilada, albúmina, producto calcio-fósforo, que en otros estudios han demostrado estar asociados al pronóstico ${ }^{32}$. Esto puede tener varias explicaciones, en primer lugar se trata de pacientes prevalentes y no incidentes, en segundo lugar

\section{REFERENCIAS}

1. Ritz E, Rychlik I, Lacateli F, Halimi S. End-stage renal failure in type 2 diabetes. A medical castastrophe of worldwide dimensions. Am J Kidney Dis 1999; 34: 795-808.

2. Rutkowaki B, Cliacalteu A, Djukanovic L, Kiss I, Kovac A, KrivoshiIev R et al. Evolution of renal replacement therapy in Central and Eastern Europe 7 years after political and economical liberation. Nephrol Dial Transplant 1998; 13: 860-4.

3. Mazzuchi N, Schwedt E, Fernández JM, Cusumano AM, Ancao MS, Poblete H et al. Latin American Registry of Dialysis and Transplantation. 1993 Annual Dialysis Data Report. Nephrol Dial Transplant 1997; 12: 2521-7. la comorbilidad puede ser tan elevada que no permita que se exprese la influencia de los factores estudiados, el número de pacientes puede ser escaso y el tiempo de observación corto.

A pesar de que este trabajo tiene la debilidad de haber tomado pacientes prevalentes, lo que no permite tener datos sobre la morbimortalidad actuarial, y de haber estudiado los pacientes de sólo un área de Santiago que puede no ser representativo del país completo. Nosotros pensamos, sin embargo, que nuestras observaciones son representativas de lo encontrado en la práctica clínica corriente, lo que está avalado por las cifras de mortalidad bruta, hipertensión arterial y dosis de diálisis semejantes a las reportadas para la población nacional en el mismo período.

Los datos presentados demuestran un inadecuado cuidado en los pacientes en etapas precoces de la diabetes, una tardía referencia al nefrólogo que obliga a la diálisis de urgencia e imposibilita la planificación de la modalidad de diálisis y de la confección del acceso vascular. Durante el periodo de la diálisis, se demuestra un pobre control de la glicemia y de las cifras de tensión arterial y una dosis insuficiente de diálisis, todo esto asociado a una elevadísima morbimortalidad. La situación mostrada refleja deficiencias en la aplicación de las guías de control y tratamiento de diabetes existentes en nuestro país, falencias en sistemas de control de calidad y en las redes de comunicación entre los niveles de atención, que deben ser corregidos.

4. Акмal M. Hemodialysis in Diabetic Patients. Am J Kidney Dis 2001; 38: 195-9.

5. UK Prospective Diabetes Study Group. Intensive blood glucose control with sulphonylureas or insulin compared with conventional treatment and risk of complications in patients with type 2 diabetes (UKPDS 33). Lancet 1998; 352: 837-53.

6. UK Prospective Diabetes Study Group. Tight blood pressure control and risk of macrovascular and microvascular complications in type 2 diabetes. UKPDS 38. Brit Med J 1998; 317: 703-13.

7. Mogensen CE. Combined high blood pressure and glucose in type 2 diabetes. Double jeopardy. Brit Med J 1998; 317: 693-4.

8. Ritz E, Stefanski A. Diabetic nephropathy in type II diabetes. Am J Kidney Dis 1996; 27: 167-94. 
9. Jones C, Krolewsini AS, Rogus J, Xue JL, Collins A, WARRAM JH. Epidemic of end-stage renal disease in people with diabetes in the United States population. Do we know the cause? Kidney Int 2005; 67: 1684-91.

10. Kikkawa R, Arimuera T, Haneda M, Nishio T, SaWada K, Yagisawa M, Shigeta Y. Current status of type 2 (noninsulin-dependent) diabetic subjects on dialysis therapy in Japan. Diabetology 1993; 36: 1105-8.

11. Sunagawa H, Iseki K, Nishime K, Ueahara H, Toma $S$, Kinjo K, Fukiyama K. Epidemiologic analysis of diabetic patients on chronic dialysis. Nephron 1996; 74: 361-6.

12. Ministerio de Salud 2005. Clínicas GES 2005. Diabetes Mellitus Tipo 2. Disponible en: www.minsal.cl/ic/guias clínicas/diabetes (consultado en el año 2005).

13. Karnosfsky DA, Burchenal JH. The clinical evaluation of chemotherapeutic trials. Columbia Press 1949; 191-205.

14. Daugirdas J, Blake P. Handbook of dialysis (Fourth edition). En: Part. II, cap. 3, Blood-Based Therapies. Philadelphia: Editorial A. Lippincott Williams \& Wilkins, 40-45.

15. Poblete H. XXVI Cuenta de hemodiálisis crónica (HDC) en Chile. Sociedad Chilena de Nefrología. Registro de diálisis 2006; 26: 1-100.

16. Poblete H. XX Cuenta de hemodiálisis crónica (HDC) en Chile. Sociedad Chilena de Nefrología. Registro de diálisis 2000; 20: 3-42.

17. Carrasco E, Pérez-Bravo F, Santos Jl, López G, Calvillan M, Wolff C et al. One of the lowest validated incidence rates of insulin dependent Diabetes Mellitus in the Americas: Santiago, Chile. Diab Res Clin Pract 1996; 34(1001): S153-7.

18. Jadue L, Vega J, Escobar MC, Delgado B, Garrido C, Lastra P Et al. Factores de riesgo para las enfermedades no transmisibles: metodología y resultados globales de la encuesta de base CARMEN. Rev Méd Chile 1999; 127: 104-13.

19. LARON Z. Interplay between heredity and environment in recent explosion of Type 1 childhood Diabetes Mellitus. Am J Med Genet 2002; 115: 4-7.

20. Wild S, Roglic G, Green A, Sicree R, King H. Global prevalence of diabetes. Estimates for the year 2000 and projections for 2030. Diabetes Care 2004; 27: 1047-553.
21. Toscana DJ, Ross Morton, A Toffelmine FB, HoLLEND DC. Adequacy of glycemic control in hemodialysis patients, with diabetes. Diabetes Care 2006; 29: 2247-51.

22. Adler IA, Stevens RJ, Manley Se, Bilous RW, Cull CA, Holman RR, on Behalf of the UKPDS Group. Development and progression of nephropathy in type 2 diabetes: The United Kingdom Prospective Diabetes Study (UKPDS 64). Kidney Int 2003; 63: 225-32.

23. Ratcliff PJ, Phillips RE, Oliver DO. Late referral for maintenance dialysis. Brit Med J 1984; 288: 441-3.

24. Ifudu O, Dawood M, Homel P, Friedman EA. Excess mortality in patients starting uremia therapy without prior care by a nephrologist. Am J. Kidney Dis 1996; 28: 841-6.

25. Innes P, Rowe PA, Burden RP, Morgan AG. Early deaths on renal replacement therapy: The need for early nephrological referral. Nephrol Dial Transplant 1992; 7: 467-71.

26. Daly C, Khan I, Ramsay C, Catto G, Edwards N, MACLEOD A. Failure to plan for first dialysis prejudices survival in patients with ESRD. J Am Soc Nephrol 1995; 6(abst.): 526.

27. Oоmichi T, Еmoto M, Tabata T, Morioka T, TsujimoTO Y, TAhara H eT al. Impact of glycemic control on survival of diabetic patients on chronic regular hemodialysis. A 7 years observational study. Clinical care/education/nutrition 2006; 29: 1496500.

28. SNYDER RW, BERNS JS. Use of insulin and oral hypoglycemic medications in patients with diabetes mellitus and advanced kidney disease. Semin Dial 2004; 17: 365-70.

29. Vukusich A, Fierro A, Morales J, Fantuzzi A, Vukusich C, Mañalich J et al. Epidemiología de la hipertensión en hemodiálisis. Rev Méd Chile 2002; 130: 610-15.

30. Depner T, Dauguirdas J. Hemodialysis adecuacy 2006 (KDOQI). Am J Kidney 2006; 1: S2-589.

31. Narayan KM, Boyle JP, Thomson TJ, Stephen S, Williamson D. Lifetime risk for diabetes mellitus in the United States. JAMA 2003; 14: 1884-90.

32. Chantrel F, Enache I, Boulller M, Kolb I, Kunz K, Petitjean P et al. Abysmal prognosis of patients with type 2 diabetes entering dialysis. Nephrol Dial Transplant 1999; 14: 129-36. 